\title{
Relationship between Human Mammaglobin mRNA in Peripheral Blood and Overall Survival in Breast Cancer Patients: A Systematic Review and Meta-Analysis
}

Hyun Jung Kim¹, Hyeong Sik Ahn', II Min Ahn ${ }^{1,2}$, Gyeong-Won Lee ${ }^{3}$, Eun-Ha Koh* and Dawon Kang ${ }^{5 *}$

${ }^{1}$ Department of Preventive Medicine, College of Medicine, Korea University, Seoul, Korea

${ }^{2}$ Department of Literary Arts, Brown University, Rhode Island, USA

${ }^{3}$ Departments of Internal Medicine, Institute of Health Sciences, Gyeongsang National University, South Korea

${ }^{4}$ Departments of Laboratory Medicine, Institute of Health Sciences, Gyeongsang National University, South Korea

${ }^{5}$ Departments of Physiology, Institute of Health Sciences, Gyeongsang National University, South Korea

\begin{abstract}
Background: Breast cancer remains a leading cause of cancer-related death in women. Numerous studies have demonstrated that mammaglobin is one of the most promising markers for diagnosis of breast cancer. However, little is known about the relationship between mammaglobin and overall survival. The small number of published studies has yielded controversial results.
\end{abstract}

Objective: This study was performed to clarify the association between expression of mammaglobin in peripheral blood and prognosis in breast cancer patients.

Methods: We systematically reviewed and meta-analyzed the data showing the association between expression of mammaglobin in peripheral blood and prognosis in breast cancer patients. The data were obtained from analysis of disease free survival (DFS)/event free survival (EFS) and overall survival (OS).

Results: Our analysis showed that patients expressing mammaglobin in peripheral blood before the removal of the primary tumors or chemotherapy showed shorter DFS/EFS, but no change in OS. The detection of mammaglobin mRNA in peripheral blood was not associated with worse outcome for OS, but the effect size was not small.

Conclusion: These data indicate that a meaningful association of mammaglobin might be found if large scale studies are performed. Large prospective studies are necessary to determine the prognostic value of the detection of mammaglobin mRNA in peripheral blood of breast cancer patients.

Keywords: Breast neoplasms; Disease-free survival; SCGB2A2 protein; Survival

Abbreviations: CI: Confidence Interval; DFS: Disease Free Survival; EFS: Event Free Survival; ER: Estrogen Receptor; HR: Hazard Ratio; OS: Overall Survival; RR: Risk Ratio

\section{Introduction}

Breast cancer is one of the most frequent cancers in women and its incidence is constantly increasing. Despite many advances in early diagnostics and treatment strategies, breast cancer remains a leading cause of cancer-related death in women; approximately $40 \%$ of patients suffer death through the development of metastases [1].

It is important to detect occult metastasis in early-stage patients to provide an accurate prognosis to patients. Detection of relevant tumor biomarkers in circulating tumor cells (CTC) is consummate for detection of occult metastasis in early-stage patients. CTCs are observed in the peripheral blood of many patients although they do not show disseminated tumor cells in the bone marrow [1], nor do they represent metastasis [2-4]. CTC counts are used as a prognostic factor for overall survival (OS) in cancer patients [5]. Detection of molecular markers specific to cancer cells or tissues in CTC could enhance prognostic information in breast cancer patients. However, several markers identified in CTC obtained from breast cancer patients, such as cytokeratin, CEA, mucin 1, EpCAM and human epidermal growth factor receptor $2[1,6]$ lack sufficient sensitivity and specificity for detecting breast cancer CTC [7], with the exception of mammaglobin (SCGB2A2), which is a member of the uteroglobin protein family [8-10].
Mammaglobin is specifically expressed in the mammary epithelium and overexpressed in breast cancer. It is not expressed in CTCs obtained from healthy donors [9,11-17] or from benign breast disease $[18,19]$. However, detection frequency of mammaglobin in blood varies from $10 \%[15,17,18]$ to $>35 \%[20-22]$ of patients with breast cancer. Increased mammaglobin expression in breast cancer was associated with clinical and biologic features characteristic of a less aggressive phenotype as expression was significantly higher in estrogen receptor (ER)-positive tumors $[23,24]$. A small number of reports has shown that expression of mammaglobin affects disease (or event) free survival (DFS or EFS) and OS, in agreement with numerous studies showing that mammaglobin is one of the most promising markers for breast cancer diagnosis [25-30]. However, the relationship between mammaglobin and OS,

*Corresponding author: Eun-Ha Koh, Department of Laboratory Medicine, Institute of Health Sciences, Gyeongsang National University, 90 Chilam, Jinju 660-751, South Korea, Tel: 82-55-750-8678; Fax: 82-55-772-8049; E-mail: ehkohmd@gnu.ac.kr

Dawon Kang, Department of Physiology, Institute of Health Sciences, College of Medicine, Gyeongsang National University, 15 Jinju-daero 816 beon-gil, Jinju 52727 South Korea, Tel: 82-55-772-8044; Fax: 82-55-772-8049; E-mail: dawon@gnu.ac.kr

Received August 16, 2017; Accepted August 21, 2017; Published August 28 2017

Citation: Kim HJ, Ahn HS, Ahn IM, Lee W, Koh EH, et al. (2017) Relationship between Human Mammaglobin mRNA in Peripheral Blood and Overall Survival in Breast Cancer Patients: A Systematic Review and Meta-Analysis. J Oncol Transl Res 3: 117. doi: 10.4172/2476-2261.1000117

Copyright: (c) $2017 \mathrm{Kim} \mathrm{HJ}$, et al. This is an open-access article distributed under the terms of the Creative Commons Attribution License, which permits unrestricted use, distribution, and reproduction in any medium, provided the original author and source are credited. 
a gold standard primary end point to evaluate the outcome of any drug, biologic, intervention or procedure that is assessed in oncologic clinical trials [31], is controversial among researchers. Some reports demonstrate that high expression of mammaglobin in peripheral blood decreases OS of breast cancer patients and some reports find the opposite [25-30].

This systematic review was performed to determine whether expression of mammaglobin in the peripheral blood affects OS. Based on meta-analysis, the expression of mammaglobin insignificantly affects OS, but size effect is high, suggesting that mammaglobin could be a prognostic marker for breast cancer.

\section{Methods}

The aim of this systematic review and meta-analysis was to compare OS of breast cancer patients with and without mammaglobin expression. We followed the Cochrane review method [32] and reported our results according to the Preferred Reporting Items for Systematic Reviews and Meta-Analyses (PRISMA) statement [33].

\section{Data Source and Literature Source (Literature Search Strategy)}

In May 2014, we searched the following general biomedical and science database: MEDLINE, EMBASE, the Cochrane Central Register of Controlled Trials (CENTRAL) and KoreaMed. Our search strategies were developed in collaboration with systematic review methodology experts. Search strategies were tailored to each database using the following keywords: breast cancer, mammaglobin, blood and prognosis (Supplementary Table 1). We also manually searched reference lists of all included publications and clinical trial registries (http://www. ClinicalTrial.gov) for ongoing studies. Our searches were not limited by publication time or language.

\section{Study Selection}

The inclusion of all studies was blindly determined by two reviewers (Kang and Koh) according to the pre-specified selection criteria. Two reviewers screened each title and abstract of identified studies. After first selection, full texts were reviewed. Studies were included in our meta-analysis if they cover breast cancer, mammaglobin, OS and peripheral blood.

\section{Data Extraction}

Relevant data were independently extracted by two reviewers using a predefined data extraction form. We resolved any discrepancy by discussion. The following variables were extracted from studies: hazard ratio (HR) or risk ratio (RR), standardized error with $95 \%$ confidence interval (CI), origin of population, stages of cancer, mean age of patients, mean length of follow-up month and percentage of mammaglobin expression in patients. If the above variables were unclear in the studies, we attempted to contact authors of the original article for the data via email.

\section{Assessment of Methodological Quality}

Two reviewers (Kang and Koh) independently evaluated the methodological qualities for identified studies using the Quality In Prognosis Studies (QUIPS) tool [34]. Any unresolved disagreements were resolved through discussion or the review from the third reviewer (HJ KIM). Publication bias was not assessed. Tests for funnel plot asymmetry are only conducted when at least 10 studies are included in the meta-analysis. This analysis includes only 6 studies.

\section{Statistical Analysis}

The main outcome of this meta-analysis was analysis of the effect of mammaglobin on survival (DFS, EFS and OS) in breast cancer patients. The most relevant statistic to use for meta-analyses of time-to-event is the HR. If reported in each article, HR was used in our meta-analysis, in several of the identified studies; the RR was presented rather than a timeto-event analysis of these events. Therefore, we separately analyzed studies using hazard ratios and relative risk ratios. We analyzed survival data using estimating $\log (\mathrm{HR})$ and $\operatorname{Var}(\log (\mathrm{HR}))$ from published summary statistics to combine trials that measure the same statistics but use different methods. The 95\% CI were reported on all estimates. For heterogeneity, we calculated the $\mathrm{I}^{2}$ statistic to describe the degree of heterogeneity with value of above $50 \%$ considered high. All cases were analyzed through the random-effect model as clinical heterogeneity existed even if statistical heterogeneity did not. To explore variation between trials, we conducted subgroups analyses by types of analysis (multivariate and univariate). We used RevMan version 5.2 for these analyses.

\section{Results}

\section{Study selection}

Searches of the databases resulted in 601 articles (Figure 1). Of these, 594 publications were excluded as it was clear from the title and abstract that they did not meet the selection criteria. For the remaining 7 articles, we obtained full manuscripts, and following scrutiny of these, we identified 6 potentially relevant studies; one publication was excluded because of unclear criteria. The publication was divided into groups termed Test+ and Test-. Test+ meant that patients had either a bone marrow or a peripheral blood test positive for mammaglobin or carcinoembryonic antigen [35]. Therefore, the total number of studies included in the review was 6 (Figure 1).

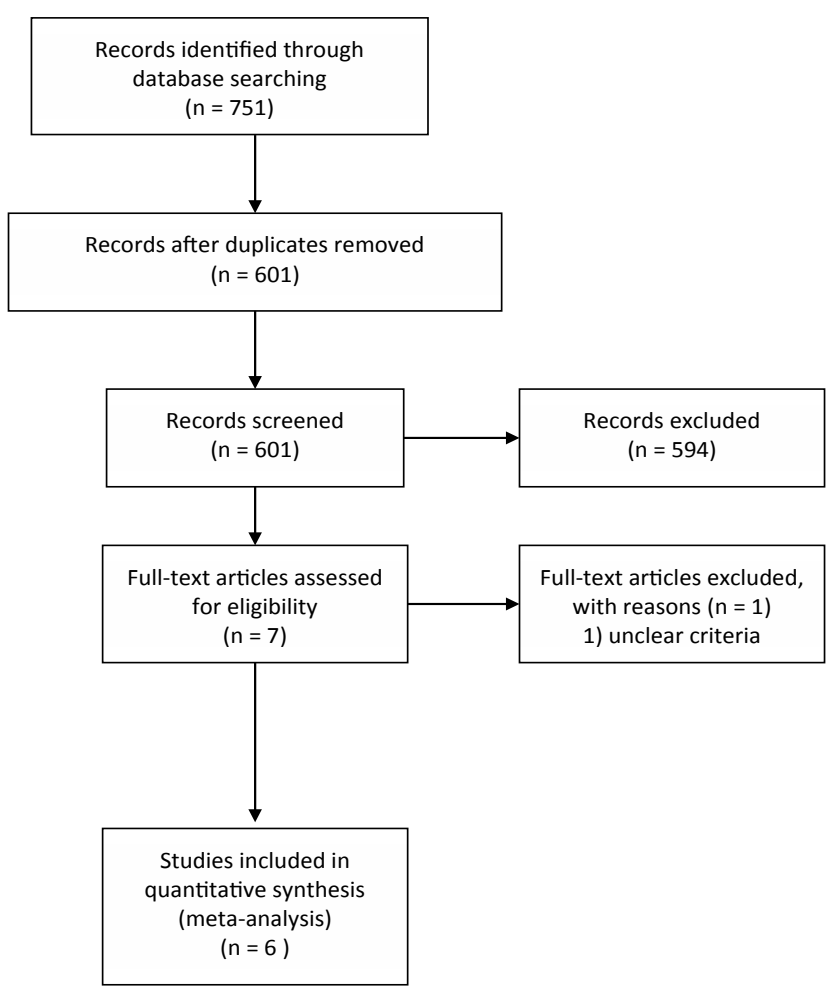

Figure 1: Flow diagram of study selection. 
Citation: Kim HJ, Ahn HS, Ahn IM, Lee W, Koh EH, et al. (2017) Relationship between Human Mammaglobin mRNA in Peripheral Blood and Overall Survival in Breast Cancer Patients: A Systematic Review and Meta-Analysis. J Oncol Transl Res 3: 117. doi: 10.4172/2476-2261.1000117

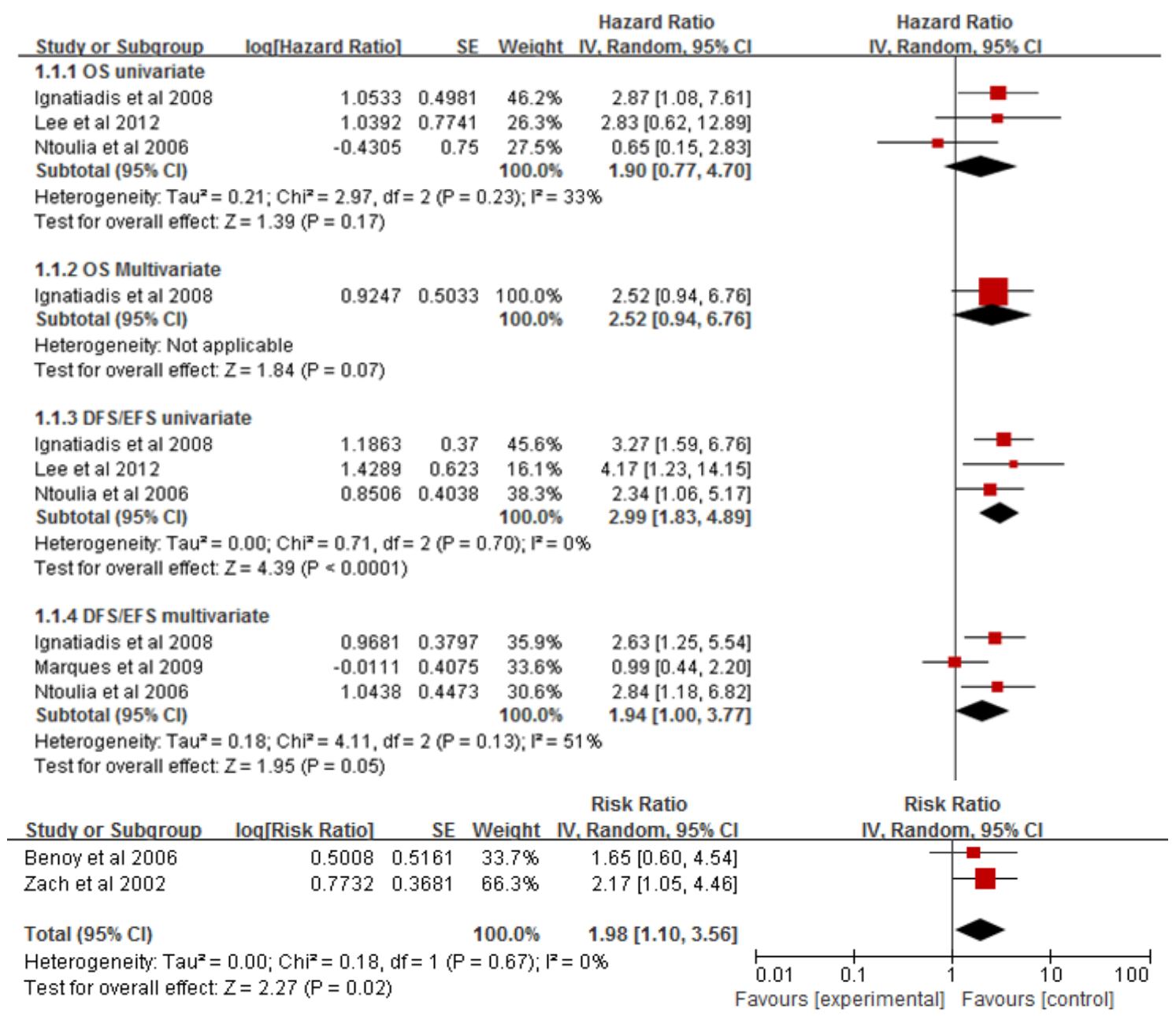

Figure 2: Forrest plots and meta-analysis of studies assessing hazard ratio (A) and risk ratio (B) of mammaglobin-positive group as compared to mammaglobinnegative group in peripheral blood. Survival data are reported as (1.1.1-2) overall survival (OS) and (1.1.3-4) disease-free survival (DFS).

\begin{tabular}{|c|c|c|c|c|c|c|}
\hline Authors & Origin of population & $\begin{array}{l}\text { Number of } \\
\text { control }\end{array}$ & $\begin{array}{l}\text { Number of } \\
\text { patients }\end{array}$ & Mean age & $\begin{array}{l}\text { Mean length of follow-up, } \\
\text { month }\end{array}$ & MAM expression ( $\%$, positive) \\
\hline Benoy et al. [25] & Belgium & 37 & 148 & $27-88$ & 25.8 & 19.6 \\
\hline Ignatiadis et al. [30] & Greece & - & 175 & $\begin{array}{c}54 \\
(28-75)\end{array}$ & 60.0 & 8.0 \\
\hline Lee et al. [26] & Korea & 16 & 82 & $\begin{array}{c}50 \\
(26-82)\end{array}$ & 20.9 & 48.8 \\
\hline Marques et al. [28] & Portugal & 28 & 321 & $\begin{array}{c}51 \\
(22-81)\end{array}$ & 43.0 & 18.7 \\
\hline Ntoulia et al. [27] & Greece & 70 & 140 & $\begin{array}{c}53 \\
(26-75)\end{array}$ & 24.0 & 15.0 \\
\hline Zach et al. [29] & Austria & 143 & 467 & $\begin{array}{c}56 \\
(26-94)\end{array}$ & 19.0 & 34 \\
\hline
\end{tabular}

Table 1: Summary of studies included in the meta-analysis.

\section{Study Characteristics and Patient Populations}

The characteristics of the six studies included in the meta-analysis are summarized in Table 1. The mean age of participant ranged from 50 to 56 years; one study did not report mean age of patients [25]. The mean length of follow-up ranged from 19.0 to 60.0 months. The percentage of mammaglobin expression in peripheral blood ranged $8.0 \%$ to $48.8 \%$ in breast cancer patients. Four studies included sample obtained from patients with metastatic breast cancer [25-27,29].

\section{Quality of the Included Studies}

The results of the quality assessment indicated that all six studies were high quality. As shown in Table 2, most studies included in the meta-analysis showed risk of bias judged as 'low' in six assessments (study participation, study attrition, prognostic factor measurement, outcome measurement and statistical analysis and presentation). However, in the outcome measurement and statistical analysis, Marques et al. and Zach et al. showed moderate risk of bias [28,29], because there 
Citation: Kim HJ, Ahn HS, Ahn IM, Lee W, Koh EH, et al. (2017) Relationship between Human Mammaglobin mRNA in Peripheral Blood and Overall Survival in Breast Cancer Patients: A Systematic Review and Meta-Analysis. J Oncol Transl Res 3: 117. doi: 10.4172/2476-2261.1000117

Page 4 of 6

\begin{tabular}{|l|c|c|c|c|c|c|}
\hline Prognosis & \multicolumn{3}{|c|}{ Risk of bias } \\
\hline Study ID & Study Participation & $\begin{array}{c}\text { Study } \\
\text { Attrition }\end{array}$ & $\begin{array}{c}\text { Prognostic Factor } \\
\text { Measurement }\end{array}$ & $\begin{array}{c}\text { Outcome Measurement } \\
\text { Study Confounding } \\
\text { and Presentation }\end{array}$ & Low \\
\hline Benoy et al. [25] & Low & Low & Low & Low \\
\hline Ignatiadis et al. [30] & Low & Low & Low & Low & Low \\
\hline Lee et al. [26] & Low & Low & Low & Moderate & Low \\
\hline Marques et al. [28] & Low & Low & Low & Low & Low \\
\hline Ntoulia et al. [27] & Low & Low & Low & Low & Mow \\
\hline Zach et al. [29] & Low & Low & Low & Low & Low \\
\hline
\end{tabular}

EFS: Event Free Survival; HR: Hazard Ratio; OS: Overall Survival; RR: Risk Ratio

Table 2: Risk of bias of studies included in the meta-analysis.

was no HR when displaying OS data [28] and Zach et al. reported OS data with RR other than HR. In addition, they did not show EFS data [29]. We judged risk of bias in the assessment of study confounding as moderate because OS data were analyzed by only univariate analysis [25-27].

\section{Correlation between Mammaglobin and DFS/EFS}

Of the six publications, four studies analyzed DFS/EFS using univariate and/or multivariate analyses [26-28,30]. Univariate analysis showed shorter DFS/EFS in patients with expression of mammaglobin in peripheral blood than in patients without expression of mammaglobin $[26,27,30]$. There was statistically significant association between detection of mammaglobin in peripheral blood and DFS/EFS $(\mathrm{HR}=2.99,95 \% \mathrm{CI}=1.83-4.89, \mathrm{P}<0.0001)$. There was no statistically significant heterogeneity between studies $\left(\mathrm{I}^{2}=0 \%\right)$. Multivariate analysis showed that there was a trend that detection of mammaglobin in peripheral blood is associated with short DFS/EFS $(\mathrm{HR}=1.94,95 \% \mathrm{CI}=1.00-3.77, \mathrm{P}=0.05)[27,28,30]$. There was moderate statistical heterogeneity between studies $\left(\mathrm{I}^{2}=51 \%, \mathrm{P}<0.13\right)$.

\section{Relation between Expression of Mammaglobin and OS}

Five publications analyzed the relationship between mammaglobin expression and OS using univariate and/or multivariate analyses. In the analysis of OS, three studies used HR [26,27,30], while two studies used RR [25,29]. The pooled univariate analysis did not show a significant difference in OS between mammaglobin-positive group and mammaglobin-negative group in peripheral blood (HR=1.90; 95\% $\mathrm{CI}=0.77-4.70, \mathrm{P}=0.17)$. There was no statistical heterogeneity between studies $\left(\mathrm{I}^{2}=33 \%\right)$. Multivariate analysis also showed insignificant relationship between mammaglobin expression and $\mathrm{OS}(\mathrm{HR}=2.52$; 95\% CI=0.94-6.76, $\mathrm{P}=0.07$ ) [30]. The two studies using RR showed that there was statistically significant association between expression of mammaglobin in peripheral blood and OS. ( $R R=1.98 ; 95 \% \mathrm{CI}=1.10$ $3.56, \mathrm{P}=0.02)$. There was no statistical heterogeneity between studies $\left(\mathrm{I}^{2}=0 \%\right)$.

\section{Discussion}

This systematic review and meta-analysis were performed to clarify the association between mammaglobin expression in peripheral blood and prognosis in breast cancer patients by analyzing DFS/EFS and OS. This study focused on OS, which is universally recognized as being unambiguous, unbiased, with a defined end point of paramount clinical relevance; positive results provide confirmatory evidence that a given treatment extends the life of a patient [31].

Patients expressing mammaglobin in peripheral blood before the removal of the primary tumors or chemotherapy showed significantly shorter DFS/EFS as judged by univariate analysis. In addition, multivariate analysis exhibited a trend that mammaglobin expression decreased DFS/EFS. Two studies included in multivariate analysis demonstrated that the detection of peripheral blood mammaglobin positive cells reduced disease-free interval (DFI) and DFS in women with early breast cancer $[27,30]$, whereas another study did not find that mammaglobin expression predicted poor DFS [28]. In multivariate analysis, the studies showed heterogeneity among them unlike data obtained by univariate analysis. The differences are likely to result from a variety of factors including origin of population and the number of patients analyzed. The first two studies targeted Greeks patients and included five identical authors in each study $[27,30]$. The other study targeted the Portuguese population and included more patients than other two studies [28].

Unlike correlation between mammaglobin and DFS/EFS, the expression of mammaglobin in peripheral blood was not associated with worse outcome for OS in either univariate or multivariate analyses $[26,27,30]$. The data were statistically insignificant, but effect size was not small, indicating that a meaningful association of OS with mammaglobin may be found if large scale studies are performed. This insignificant relationship between mammaglobin and OS is driven by results from the study by Ntoulia et al. [27], among the studies analyzed. Ntoulia et al. [27] reported that operable breast cancer patients (stage I-II) without mammaglobin expression in peripheral blood showed worse OS compared with mammaglobin-positive patients, although there was no significance between them $(\mathrm{P}=0.566)$. These results may be explained by the fact that an absence of mammaglobin in blood samples does not mean the absence of CTC; that is, CTM in blood of mammaglobin-negative patients would not be detected by mammaglobin-specific primers. In addition, the study included fewer breast cancer patients expressing ER than the other two studies. These studies used HR as a variable, but analysis of RR demonstrated significant correlation between mammaglobin expression and OS in the other two studies $[25,29]$. Breast cancer patients who were mammaglobin-negative at time of diagnosis of metastasis lived longer than mammaglobin-positive patients.

From this systematic review and meta-analysis, we found a trend that mammaglobin detection in peripheral blood correlates with poor outcome. However, this review has some limitations. First, the number of studies and the number of patients included are relatively small. The patient populations vary across studies, which may cause heterogeneity within our analysis. Five out of six studies were carried out targeting European patients with breast cancer. Ethnic differences can lead to different results. Racial/ ethnic differences result in survival differences in breast cancer patients 
[36]. Second, publication bias and selective reporting biases cannot be excluded. Finally, the studies analyzed for this review used different cutoff values for detection of mammaglobin. Although we use same cutoff, the ratio of detection can be potentially different depending on instruments, materials and researchers. In particular, the differences in sensitivity and efficiency of primers used for RT-PCR or real-time PCR could cause differences in the results of each study.

In conclusion, our study provides evidence that the detection of mammaglobin mRNA in peripheral blood before the removal of a primary tumor or the initiation of chemotherapy is a prognostic factor for shorter DFS. The detection of mammaglobin mRNA in peripheral blood was not associated with worse outcome for OS, but effect size was not small. Therefore, large prospective studies are necessary to determine the prognostic value of the detection of mammaglobin mRNA in peripheral blood of breast cancer patients.

\section{Conflicts of Interest}

We have no financial or commercial conflicts of interest in this study.

\section{Source of Funding}

This work was supported by a grant from the Basic Science Research Program through the National Research Foundation (NRF) of Korea funded by the Ministry of Science, ICT and Future Planning (2015R1A5A2008833).

\section{References}

1. Lacroix M (2006) Significance, detection and markers of disseminated breast cancer cells. Endocr Relat Cancer 13: 1033-1067.

2. Cristofanilli M, Hayes DF, Budd GT, Ellis MJ, Stopeck A, et al. (2005) Circulating tumor cells: A novel prognostic factor for newly diagnosed metastatic breas cancer. J Clin Oncol, pp: 231420-1430.

3. Gaforio JJ, Serrano MJ, Sanchez-Rovira P, Sirvent A, Delgado-Rodriguez, et al. (2003) Detection of breast cancer cells in the peripheral blood is positively correlated with estrogen-receptor status and predicts for poor prognosis. Int $J$ Cancer 107: 984-990.

4. Muller V, Stahmann N, Riethdorf S, Rau T, Zabel T, et al. (2005) Circulating tumor cells in breast cancer: correlation to bone marrow micrometastases, heterogeneous response to systemic therapy and low proliferative activity. Clin Cancer Res 11: 3678-3685.

5. Goldkorn A, Ely B, Quinn DI, Tangen CM, Fink LM, et al. (2014) Circulating tumor cell counts are prognostic of overall survival in SWOG S0421: A phase III trial of docetaxel with or without atrasentan for metastatic castration-resistan prostate cancer. J Clin Oncol 32: 1136-1142.

6. Mikulova V, Kolostova K, Zima T (2011) Methods for detection of circulating tumour cells and their clinical value in cancer patients. Folia Biol (Praha) 57: 151-161.

7. Suchy B, Austrup F, Driesel G, Eder C, Kusiak I, et al. (2000) Detection of mammaglobin expressing cells in blood of breast cancer patients. Cancer Lett 158: $171-178$.

8. Lin H, Balic M, Zheng S, Datar R, Cote RJ (2011) Disseminated and circulating tumor cells: Role in effective cancer management. Crit Rev Oncol Hematol 77 : $1-11$.

9. Corradini P, Voena C, Astolfi M, Delloro S, Pilotti S, et al. (2001) Maspin and mammaglobin genes are specific markers for RT-PCR detection of minimal residual disease in patients with breast cancer. Ann Oncol 12: 1693-1698.

10. Ghersevich S, Ceballos MP (2014) Mammaglobin A: review and clinical utility Adv Clin Chem 64: 241-268.

11. Watson MA, Fleming TP (1996) Mammaglobin, a mammary-specific member of the uteroglobin gene family, is overexpressed in human breast cancer. Cancer Res 56: 860-865.

12. Fleming TP, Watson MA (2000) Mammaglobin, a breast-specific gene and its utility as a marker for breast cancer. Ann N Y Acad Sci 923: 78-89.

13. Ceballos MP, Zumoffen C, Massa E, Cipulli G, Funes CC, et al. (2011) Detection of mammaglogin $A$ in blood from breast cancer patients, before and after treatment, using a one-tube nested PCR protocol. Association with the absence of tumor estrogen receptors. Clin Biochem 44: 1429-1433.
14. O'Brien N, Maguire TM, O'Donovan N, Lynch N, Hill AD, et al. (2002) Mammaglobin A: A promising marker for breast cancer. Clin Chem 48: $1362-$ 1364.

15. Mercatali L, Valenti V, Calistri D, Calpona S, Rosti G, et al. (2006) RT-PCR determination of maspin and mammaglobin $B$ in peripheral blood of healthy donors and breast cancer patients. Ann Oncol 17: 424-428.

16. Silva AL, Tome MJ, Correia AE, Passos-Coelho JL (2002) Human mammaglobin RT-PCR assay for detection of occult breast cancer cells in hematopoietic products. Ann Oncol 13: 422-429.

17. Ballestrero A, Garuti A, Bertolotto M, Rocco I, Boy D, et al. (2005) Effect of different cytokines on mammaglobin and maspin gene expression in normal leukocytes: possible relevance to the assays for the detection of micrometastatic breast cancer. $\mathrm{Br} \mathrm{J}$ Cancer 92: 1948-1952.

18. Ferro P, Franceschini MC, Bacigalupo B, Dessanti P, Falco E, et al. (2010) Detection of circulating tumour cells in breast cancer patients using human mammaglobin RT-PCR: association with clinical prognostic factors. Anticancer Res 30: 2377-2382.

19. Roncella S, Ferro P, Bacigalupo B, Pronzato P, Tognoni A, et al. (2005) Human mammaglobin mRNA is a reliable molecular marker for detecting occult breas cancer cells in peripheral blood. J Exp Clin Cancer Res 24: 265-271.

20. Zach O, Kasparu H, Krieger O, Hehenwarter W, Girschikofsky M, et al. (1999) Detection of circulating mammary carcinoma cells in the peripheral blood of breast cancer patients via a nested reverse transcriptase polymerase chain reaction assay for mammaglobin mRNA. J Clin Oncol 17: 2015-2019.

21. Cerveira N, Torres L, Rocha P, Bizarro S, Pereira D, et al. (2004) Highly sensitive detection of the MGB1 transcript (mammaglobin) in the peripheral blood of breast cancer patients. Int J Cancer 108: 592-595.

22. Zehentner BK, Persing DH, Deme A, Toure P, Hawes SE, et al. (2004) Mammaglobin as a novel breast cancer biomarker: Multigene reverse transcription-PCR assay and sandwich ELISA. Clin Chem 50: 2069-2076.

23. Nunez-Villar MJ, Martinez-Arribas F, Pollan M, Lucas AR, Sanchez J, et al (2003) Elevated mammaglobin (h-MAM) expression in breast cancer is associated with clinical and biological features defining a less aggressive tumour phenotype. Breast Cancer Res 5: R65-70.

24. Span PN, Waanders E, Manders P, Heuvel JJ, Foekens JA, et al. (2004) Mammaglobin is associated with low-grade, steroid receptor-positive breast tumors from postmenopausal patients, and has independent prognostic value for relapse-free survival time. J Clin Oncol 22: 691-698.

25. Benoy IH, Elst H, Philips M, Wuyts H, Van Dam P, et al. (2006) Real-time RT-PCR detection of disseminated tumour cells in bone marrow has superior prognostic significance in comparison with circulating tumour cells in patients with breast cancer. $\mathrm{Br} \mathrm{J}$ Cancer 94: 672-680.

26. Lee GW, Kim JY, Koh EH, Kang D, Choi DS, et al. (2012) Plasma human mammaglobin mRNA associated with poor outcome in patients with breast cancer. Genet Mol Res 11: 4034-4042.

27. Ntoulia $M$, Stathopoulou $A$, Ignatiadis $M$, Malamos $N$, Mavroudis $D$, et al. (2006) Detection of Mammaglobin A-mRNA-positive circulating tumor cells in peripheral blood of patients with operable breast cancer with nested RT-PCR Clin Biochem 39: 879-887.

28. Marques AR, Teixeira E, Diamond J, Correia H, Santos S, et al. (2009) Detection of human mammaglobin mRNA in serial peripheral blood samples from patients with non-metastatic breast cancer is not predictive of disease recurrence. Breast Cancer Res Treat 114: 223-232.

29. Zach O, Kasparu H, Wagner H, Krieger O, Lutz D (2002) Prognostic value of tumour cell detection in peripheral blood of breast cancer patients. Acta Med Austriaca Suppl 59: 32-34.

30. Ignatiadis M, Kallergi G, Ntoulia M, Perraki M, Apostolaki S, et al. (2008) Prognostic value of the molecular detection of circulating tumor cells using a multimarker reverse transcription-PCR assay for cytokeratin 19, mammaglobin $A$ and HER2 in early breast cancer. Clin Cancer Res 14: 2593-2600.

31. Driscoll JJ, Rixe O (2009) Overall survival: still the gold standard: Why overal survival remains the definitive end point in cancer clinical trials. Cancer $\mathrm{J} 15$ : 401-405.

32. Shuster J (2011) Review: Cochrane handbook for systematic reviews for interventions, Version 5.1.0, published 3/2011. Julian P.T. Higgins and Sally Green, Editors. Res Syn Meth 2: 126-130. 
Citation: Kim HJ, Ahn HS, Ahn IM, Lee W, Koh EH, et al. (2017) Relationship between Human Mammaglobin mRNA in Peripheral Blood and Overall Survival in Breast Cancer Patients: A Systematic Review and Meta-Analysis. J Oncol Transl Res 3: 117. doi: 10.4172/2476-2261.1000117

Page 6 of 6

33. Moher D, Liberati A, Tetzlaff J, Altman DG (2010) Preferred reporting items for systematic reviews and meta-analyses: The PRISMA statement. Int J Surg 8: 336-341.

34. Hayden JA, van der Windt DA, Cartwright JL, Cote P, Bombardier C (2013) Assessing bias in studies of prognostic factors. Ann Intern Med 158: 280286
35. Fortunato L, Mascaro A, Baldi A, Farina M, Cortese G, et al. (2009) Positive bone marrow biopsy is associated with a decreased disease-free survival in patients with operable breast cancer. Ann Surg Oncol 16: 3010-3019.

36. Boyer-Chammard A, Taylor TH, Anton-Culver H (1999) Survival differences in breast cancer among racial/ethnic groups: A population-based study. Cancer Detect Prev 23: 463-473. 